\section{THE DETECTION OF MORPHINE IN THE URINE AS A MEANS OF DIAGNOSIS.1}

BY BENNETT F. DAVENPORT, M.D., BOSTON.

IT has continued to this day a disputed question, in spite of the many exact accounts of instances when it had been done, whether the presence of morphine could actually be determined in the animal organism, and how far it could accurately be done. There has been two opposite opinions held upon the subject. According to the one, morphia, when once absorbed into the tissues, became decomposed, and could, therefore, no longer be determined as such, while the others held that it was excreted in the urine still undecomposed.

The first method for the determination of the morphine originated with Lassaigne in 1824. He, however, was enabled to detect but traces of it in the blood of horses into whose jugular vein he had injected it in large doses. Christison, in 1831, and Taylor in 1862, sought in vain to detect morphine. Clocka, in 1866 , could detect no trace of morphine in the blood of one with the opium habit. Erdman, in 1862, failed to detect morphine in the urine of several rabbits that he had poisoned with morphia. Instances of such negative results could be very numerously mentioned. They are, however, opposed by the positive results obtained by Orfila in 1839, Gscheidler in 1869 , and notably by Kauzmann and Dragendorff in 1868. These last two were enabled by their method of examination to detect the presence of morphine in the urine of those who had been poisoned by it, every time, without exception. Landsberg, however, who reviewed their method, was not able to confirm their results, for he failed in the most of his cases, and Wislicenus was not more fortunate.

Burkart, who investigated this subject still later, 1882, made use of a method combining those of Dragendorff, Kauzmann, Landsberg, and Wislicenus. His results, obtained from excreted morphine and mixtures of urine and morphine of like amount, and both injected into rabbits, made it to appear probable that the uncertain results obtained by the last two were best to be explained by the theory that either but a part of the morphine absorbed was excreted unchanged, or else that, while it was not changed as to its character, its intensity of action as a poison was. He in many cases found that none of the alkaloid was excreted unchanged into the urine. In other cases but a trace, all the rest being so changed as to prevent the usual modes of detection.

Later, Marmé, who was confirmed by Warnecke, showed that some morphine did pass unchanged into the urine, and he later devised elaborate methods for demonstrating the presence of morphine in the various fluids and tissues of the body under all possible circumstances. In 1884, Dragendorff, assisted by Schneider, published his investigations concerning the correctness of Burkart's morphine decomposition product being contained in the urine, and as to how large a portion of the morphine consumed by one having the opium habit was again excreted in the urine.

Dragendorff's method of separating morphine from the urine was as follows: The urine, without any concentration, is made slightly acid by a few drops of dilute sulphuric acid, and then shaken out with one-

${ }_{1888 .}^{1}$ Read before the Boston Society for Medical Observation, May 7, fifth one-third its volume of amylic alcohol during five minutes. It is then set one side to separate as clear as possible. This way of shaking out with fresh amylic alcohol is repeated four to five times to remove as much of the urea as possible. The fluid is then made slightly alkaline with ammonia, and the morphine itself separated by being shaken out in hot amylic alcohol. This solution in the alcohol must be most carefully freed from any of the adhering urine by settling and filtration, so that upon its evaporation no droplets of watery fluid may be formed. Repeated washings of the alcoholic morphine with water cannot be used when there is but a trace of morphine present. By his method, he claims that 0.01 grm. morphine in 300 cc. urine can be well recovered.

Of the reactions for the determining of the presence of morphine in this urine extraction, the Fröhde, that is, concent. sulphuric acid containing molybdium is the best, but it is not alone sufficient, as other alkaloidal substances give a similar red-violet coloration. Dragendorff denies the assertion of Burkart that pure morphine must give a blue-violet color with this reagent. Although pure morphine muriate does give the blue-violet color, yet this same salt, when recovered as a free alkaloid by the Dragendorff method out of a urine, will give a red-violet color. Therefore, Burkart, when from the urine of a morphineeater he obtained an alkaloid that gave a blue-violet with Fröhde's reagent, ought to have doubted its being pure morphine, but he ought to have considered it as an unaltered morphine when it gave the red-violet reaction.

To Burkart's claim that the red-violet coloring material which he obtained from the urine of a morphineeater was neither a decomposition product nor a urine compound of morphine, but an independent constituent of urine, and to his argument in support of this that he had obtained a beautiful red-violet color from the urine of a morphine-eater who had been taking it for ten days, Dragendorff replies that he has never been able to obtain from the urine of one who had not been taking morphine a constituent which either had an alkaloidal reaction or shared in the Fröhde's reaction of morphine. The blue reaction of iron chloride with morphine is of importance because that it separates morphine from all the other alkaloids which give a reaction with Fröhde's reagent similar to morphine. It is well known that the iron test requires certain special conditions: considerable purity of alkaloid, no excess of acid or reagent, and the alkaloid to be present in considerable quantity.

The experiments of Schneider confirmed the opinion of Hangmann and Marmé, that with doses of from 0.01 to 0.03 grm., morphine taken either by the mouth or under the skin, it could with certainty be discovered in the urine. His cases were those of an old woman, who had for fifteen years been a morphine-eater. At this time her doses were 0.24 to 0.36 acetate daily by the mouth. A student who was taking $0.48 \mathrm{grm}$. muriate daily, and a woman who was taking $0.024 \mathrm{grm}$. of the same daily subcutaneously. In all these cases positive results were obtained by the last-mentioned; that is, Fröhde's, the iron chloride, and also by the Huseman's reaction. A further evidence that the urine, after the use of morphine, contained that actual alkaloid, is the experiment of Burkart, who obtained from the twenty-four hours' urine of one using 1.3 to 1.55 grms. of morphine 
muriate a day an alkaloid which, injected subcutaneously into dogs and rabbits, developed the symptoms of severe morphine-poisoning. It has now been determined that from the urine of men and animals who have taken morphine either by the mouth or subcutaneously, an alkaloid can be obtained which has a crystalline form, a solubility of chemical reactions, and a physiological working like to morphine. The question whether it is actually unaltered morphine, or a decomposition product of the same, can only be answered when it has been obtained in a sufficient quantity and purity to make an ultimate elemental analysis of it possible, and for the determination of its other properties.

Among others, Scheibe and Eliassor, in 1882, have each proposed, as they claim, improvements in the Dragendorff method of separation, the former using an ethereal alcohol as his last solvent, and the latter acetic ether, with a previous precipitation of some of the constituents of the urine with lead acetate. In this last method, an intense gray or green-blue color is sometimes produced by the Fröhde's reagent, which he held to be a decomposition product of the morphine. Notta and Lugan have proposed a method similar to that of Eliassor, and he firds that the kidneys, when in their normal condition, excrete a portion of the morphine unchanged. Oxydi morphine is the name which Marmé and others have given to the form of morphine formed, as he claims, in the system in cases of chronic morphine-poisoning, and its reactions differ from morphine in certain particulars with the more generally used morphine reagents, all which he has set forth in his article, published in 1885, with most minute details. In this connection, it is of interest to note that a simple water solution of morphine seems to chauge partially in time into apomorphia, thus explaining, may be, the nausea, said to be more marked when such an old solution has been used. Furthermore, the possible influence of ptomaines upon the chemical reaction of morphine are to be considered in a medico-legal case where the sample of the alkaloid to be identified is obtained from the liver, for instance, and also from the urine after death. According to the researches of Hilger and Tamba, which were published in 1886, morphine in presence of ptomaines can be identified through its sugar and sulphuric acid reactions, whereby the violet color is sharply induced; further, also, by its like reaction with concentrated sulphuric acid on evaporation in the water-bath, with the later addition of hydrochloric acid, etc. Iodic acid may not be reduced by the morphine itself in the presence of more than a trace of ptomaine, for many ptomaines have of themselves a strong reducing action. All the other usual tests for morphine are not serviceable in the presence of ptomaines. They found, however, that boiling ether dissolved out the ptomaines from slightly acid solutions, especially if it had been evaporated upon gypsum. In this the morphine would remain undissolved. Also that in ethereal solutions of alkaloids and ptomaines, the addition of ether saturated with oxalic acid would, on long standing, precipitate out the alkaloid as a crystalline oxalate, while the ptomaine remained wholly in solution. Furthermore, Bouchard has determined that a healthy adult man produces upon an average in twenty.four hours enough urine poison to cause death in an animal weighing $46.5 \%$ as much as himself, if it be injected into its circulation.
Dr. Harley, in his "Old Vegetable Neurotics," says that during the action of opium, so long as any hypnotic and anæsthetic influence remain, the excretions are retarded; but when simply hypnotic and stimulant, or when the person becomes habituated to its use, the secretions and excretions are abundant and free. Dr. Anstie, in his "Stimulants and Narcotics," conjectures that in fatal cases there may be a condition of hasty and profuse shedding of the epithelial cells, such as occurs in some forms of fever, and which is usually associated with diminished secretion. Dr. Edes, in his "'Therapeutic Handbook," mentions the fact that in severe opium-poisoning the urine may contain albumen and casts. Professor Huseman, in his " Toxikologie," however, claims that there is simply a retention of urine in the bladder, due to diminished sensibility, rather than any diminished secretion, for the bladder is usually found to be distended in fatal cases; that a desire to void it has usually been felt, which could not be satisfied. Headland, in his "Action of Medicines," also asserts that opium scarcely, if at all, lessens the secretion of the urine.

The experiments of Dragendorff and Kaufman showed the following: In a cat which received into its stomach a dose of $0.183 \mathrm{grm}$. morph. sulph. dissolved in water, the uriue passed ten minutes later did not yet contain any morphine. In a kitten, two hours after a subcutaneous injection of $0.03 \mathrm{grm}$, it was found in abundance. In a medium-size dog, four and a half hours after a stomach dose of 0.31 , it was found in large quantities. After an injection of 0.135 in the thigh of a cat, the elimination was not yet completed after thirty-six hours. In a cat which received 0.03 in a gelatine capsule, it was fully complete in fifty-two hours. A large shepherd dog, after several large daily doses in capsules, in fifty-four hours after its last dose of 2.007, had no longer any in its urine. Later, after a dose of 2.48, it still had some at the end of eighty-four hours, but not at the end of ninety-four. A medium-size dog taking 0.31 in solution in stomach had it still in the urine at the end of forty hours, but that passed three times during the next twelve hours had none. The same dog, after a like dose, but subcutaneous, had no alkaloid in the urine at the end of fifty hours. A man who took one-sixth grm. had it in his urine during twelve hours, but not after that. Dr. Dragendorff found that the Fröhde's reagent would show its reaction in the presence of only $0.000,005$ grm. alkaloid, the Huseman test with only $0.000,01$, while the iron chloride test required not less than one part morphine in five thousand of the solution tested.

The possibility of being able to detect with certainty the presence of morphine in the urine of one who has taken the alkaloid in but the ordinary medicinal dose is of great practical utility in the detection of the opium habit, since, as is well known, the word of those addicted is not well to be relied upon in this particular; also in hospital practice for the detection of relapses in those who have been under treatment, but have so far recovered as to be trusted with some outside liberty.

I have recently had occasion to examine five samples of urine for Dr. Goldsmith, the Superintendent of the Butler Hospital at Providence. 'Two were from patients who were thought to be cured, and had been allowed to go outside before their final discharge. As morphine was found in neither of them, they were, therefore, discharged from the hospital. Another 
was from a case which had been known to take onehalf to one and one-half grains morphine daily for some time, but had omitted it for two days, had then taken one-half grain. Beginning thirty-six hours after this last dose, the next twenty-four hours' urine gave no reaction for morphine. The next two samples were from patients taking one-eighth of a grain three times daily. In each of these morphine was found. It is, of course, needless to say that the probability of the presence or absence of morphine was unknown before it was reported upon. These are simply examples of within what limits of time and dose I have been able to detect its presence in the urine secreted.

\section{CLINICAL NOTES OF A CASE OF PERITY-} PHLITIS ; LAPAROTOMY THIRTY-TWO HOURS AFTER THE BEGINNING OF THE ATTACK ; DEA'TH ON THE THIRTY-EIGHTH DAY. 1

$$
\text { BY S. E. WYMAN, M.D. CAMBRIDGE, MASS. }
$$

IT has seemed to me to be desirable to place upon record the following notes, as a contribution to the study of operative interference in cases of perityphlitis.

It need not be said that it is a matter of deep regret that the result should have been unfavorable, when everything seemed so auspicious at the time of the operation, which was not deferred until the patient's strength had been exhausted by protracted suffering. I have never answered to my own satisfaction the question, "Why must he have died?"

July 21, 1887. C. M. R., male, a native American, has never been strong; is fourteen years old. From the second to the eighth day of life "he vomited black stuff, as nearly like blood as could be." He began to grow fleshy when nine years old, and is now a pale, anæmic, fat boy, weighing at present 145 pounds. He has never cared to do as much running as his schoolfellows did; has suffered considerably from indigestion, and, during the past two years, has had several attacks very similar to the present one, having had three within the past four weeks. Yesterday ate watermelon and blackberries, and, at three o'clock, morning, was awakened with severe abdominal pain in umbilical and right iliac region. Present attack of pain very severe, causing him to scream and toss about; was treated by his parents by hot local applications until 7 A. M., at which time $I$ saw him and obtained his history. The patient has vomited twice, small quantity, liquid contents of the stomach. Expression is anxious; abdominal tendernes.s great, especially in umbilical and right iliac region. No tympanites. Considerable dulness on percussion in right iliac region. Decubitus dorsal, with knees drawn up. Pulse, 96, temperature $98.4^{\circ}$ (mouth). He was ordered morphia with belladonna sufficient to control pain, hot applications to the bowels, and liquid diet. Was seen at 10.45 A.M., when it was learned that the abdominal pain was greater and more general, and tympanites had begun to show itself. Has vomited once. Peristalsis not so active, and abdominal walls kept motionless during respiration. Pulse 90, temperature $98.6^{\circ}$. 3.15 P. M. Has been kept quiet, with almost no complaint of pain, and much less tenderness, by the morphia. His face is flushed. Pulse,

1 Read before the Middlesex South District Medical Society, January 18,1888 .
90-120, temperature $100^{\circ}$ (mouth). Pupils not contracted. 7.12 P. M. Hourly administration of morphia has kept him free from pain and vomiting. Urine, scanty in amount, passed with some difficulty. No change in local symptoms. He has been inclined all day to lie on his right side, and twice has been found sleeping in this position, although he thinks it causes more tenderness in right iliac region. Pulse, 112 , temperature, $100.8^{\circ}$.

July $22 ; 6.45$ A. M. Passed a fairly comfortable night, and complained less of pain. There has been no vomiting; no dejection; urine rather scanty; less abdominal tenderness generally, as well as in right iliac fossa. Pulse 108 , temperature $99.6^{\circ}$ (mouth). 10.45 A. м. Was seen in consultation by Drs. Marcy and Nelson, who concurred in the diagnosis, and advised operative interference. Ether was administered and laparotomy was performed by Dr. H. O. Marcy, whose notes I take the liberty of copying. The operation lasted more than ninety minutes.

"When under ether there was to be clearly outlined under the thick abdominal wall a firm resisting thickening in the right iliac quarter. An incision was made five inches long, through an inch or more of fat, and the muscles divided. The peritoneum was adherent over a space of about two inches to the omentum and surrounding parts, and the adbesions were separated with some difficulty and hæmorrhage. The head of the colon, with the appendix. was imbedded in a thick, organized mass, and firmly adherent to the abdominal wall. The adhesions were broken up with much difficulty, and the hæmorrhage was quite troublesome. The bleeding, adherent omentum was sewed through by the double stitch with catgut, and excised. The intestine above and below was found healthy. It was clearly determined that there was no suppuration about the appendix. The plastic mass was too firmly adherent and vascular to be safely removed except a small portion between the head of the colon and the abdominal wall. The bleeding points were secured and the wound closed, the peritoneum with a fine continuous animal suture, and the external wound under the mercuric bichloride solution irrigation."

He came out of his ether well, vomited at 2.30 P.M. Pulse, 132, temperature $98.4^{\circ}$ (ax.).

$$
\text { Re Is to have morphia by suppository, p. r. n. }
$$

9.30 P. M. Has vomited once or twice, and been made comfortable by morphia suppositories. Is fed by enemata of

$$
\begin{aligned}
& \text { Murdock's Liquid Food } \\
& \text { Whiskey : } \\
& \text { Water, q. s. : }
\end{aligned}
$$$$
\begin{gathered}
: \\
: \\
\text { once in four hours. }
\end{gathered}
$$

Pulse 126-133, temperature $98.4^{\circ}$ (ax.), respiration 18. No urine passed.

July $23 ; 7$ A. M. Good night. Urine passed twice. Moderate amount of morphia given. Tongue moist and clean. Pulse 128, regular, temperature $98.4^{\circ}$, respiration 18 . 9.45 P. M. Has romited four times. Urine passed once. Has complained of soreness in region of wound. No pain. Has been very thirsty. Pulse 126 , temperature $101^{\circ}$ (m.), respiration 20 .

July 24th ; 7.30 A. M. Required morphia once in three hours. Vomited three times during night. Passed quite a large amount of urine. Flatus per anum first time this morning. Decubitus dorsal, knees erect in the form of a tent. Hands and arms over his head. Pulse 120 , temperature $99.2^{\circ}$, respiration 20 . 